\title{
Coordinated Tuning of Proportional-Integral Power System Stabilizers in Multimachine Power Systems
}

\author{
CHERN-LIN CHEN and YUAN-YIH HSU \\ Department of Electrical Engineering, National Taiwan University, Taipei (Taiwan) \\ (Received July 14, 1986)
}

\section{SUMMARY}

A new method for the coordinated synthesis of proportional-integral power system stabilizers in multimachine power systems is presented in this paper. In contrast to the previous approaches which are iterative in nature, the stabilizer constants are obtained in a single step by solving a set of algebraic equations. This makes the proposed approach much more efficient than other methods. The effectiveness of the proposed algorithm is illustrated by the simultaneous selection of the parameters of three proportional-integral stabilizers in a multimachine power system. It is shown that the eigenvalues associated with the poorly damped electromechanical modes can be exactly assigned to desired locations in a very efficient way.

\section{INTRODUCTION}

Considerable efforts have been devoted to the application of power system stabilizers (PSSs) to enhance the damping of the low frequency electromechanical oscillations in the range $0.5-2 \mathrm{~Hz}[1-12]$. The conventional PSS which is widely employed by the utilities is a lead-lag network used for improving the phase lag in the system. Several approaches to the simultaneous tuning of conventional PSS parameters in multimachine power systems have been reported in the literature [13-23]. Reference 13 is mainly concerned with the application of eigenvalue analysis to the identification of the most effective PSS locations. However, the selection of PSS parameters was not considered. A sequential procedure for determining the optimum site for stabilizer application is proposed by Abdalla et al. [14]. This proce- dure is of limited use when the oscillation mode is affected by several generators.

In ref. 15 a sequential eigenvalue assignment algorithm for selecting the parameters of stabilizers in a multimachine power system is proposed. A major disadvantage of this method is that the sequential addition of stabilizers will disturb the previously assigned eigenvalues. In order to avoid this undesirable effect of eigenvalue drift, several approaches to the simultaneous tuning of stabilizer parameters have been proposed [16-23]. Reference 16 employs the concepts of synchronizing and damping torque coefficients for stabilizer tuning. Several disadvantages arise in this approach. The system eigenvalues are not assigned exactly since the damping coefficient of the mechanical modes is specified for the inner mechanical loop of each machine rather than for the system eigenvalue. The computation of the transfer function of the stabilizers using signal flow graphs is a formidable task for a large system. Moreover, the recursive algorithm to avoid eigenvalue drift by readjusting the PSS parameters would take considerable computational time. In refs. 17 and 18, a recursive algorithm based on the component connection method is given to take into account the dynamic interactions between the machines. The algorithm relies on the computation of eigenvector matrices at each iteration which is very time consuming. A recent report [19] uses the frequency response method including eigenvalue assignment. The transfer function of the stabilizer is obtained by least square approximation. This approach is suitable only for the preliminary setting of the PSS for the stability studies. In another paper by Doi and Abe [20], linear programming and eigenvalue sensitivity are employed. The stabilizer parameters are determined by minimizing a 
performance index which is the sum of all PSS gains. This paper is based on the assumption that the change of the power swing eigenvalue and of the frequency response of the PSS in the neighbourhood of the power swing frequency are very small. This assumption is not valid all the time. Recently, Lim and Elangovan [21 - 23] proposed a frequency domain approach which is similar to that of ref. 16. Based on eigenvalue assignment and the fixed-point method, an iterative algorithm is presented to find the PSS parameters. However, the convergence of the PSS parameters has not been proved. As in the approach proposed by Gooi et al. [16], the iterative algorithm is not efficient from the computational point of view.

All the works mentioned previously were concerned with the coordinated tuning of the parameters of conventional lead-lag power system stabilizers. Recently, a new proportional-integral (PI) PSS was proposed by the authors [24]. Being relatively simple for practical implementation, the proposed PI PSS was shown to be very effective for improving the dynamic stability of the power system. However, the simultaneous determination of the parameters $K_{\mathrm{P}}$ and $K_{\mathrm{I}}$ of the PI PSS for a multimachine power system by using the suboptimal regulator approach of ref. 24 requires considerable computation time. Therefore, an efficient method for the coordinated tuning of the parameters of PI stabilizers is highly desirable.

In this paper, a new approach based on decentralized modal control (DMC) theory is proposed for the simultaneous selection of PI PSS parameters in a multimachine power system. Unlike the previous methods which are iterative in nature, stabilizer parameters are obtained in a single step by solving a set of algebraic equations which are derived by using an exact model reduction method based on DMC theory. Therefore, the convergence problem no longer exists in the proposed approach. Moreover, it is much more efficient than the commonly used iterative algorithm.

\section{PROBLEM FORMULATION}

In the design of power system stabilizers for improving the dynamic stability of a power system, linearized incremental models are usually employed $[6,7]$. Therefore, the state equation of an interconnected power system with $n$ synchronous generators can be written in the vector-matrix differential equation form

$\dot{X}(t)=\mathrm{A} X(t)+\mathrm{B} U(t)$

where $X$ is the state vector, $U$ is the control vector comprising the PSS output signals, and $\mathbf{A}$ and $\mathbf{B}$ are constant matrices. If each synchronous generator can be modelled by five state variables, the dimensions of $X, \mathrm{~A}, \mathrm{~B}$ and $U$ are $5 n \times 1,5 n \times 5 n, 5 n \times n$ and $n \times 1$ respectively.

Taking the Laplace transformation of eqn. (1), we have the state equation in the frequency domain

$s X(s)=\mathrm{A} X(s)+\mathrm{B} U(s)$

The PSSs usually employed by the utilities are decentralized output feedback stabilizers. In other words, only the local signals in the specific generator to be equipped with the PSS can be employed as the input signal to the PSS [1 - 4]. Therefore, the control vector can be expressed in the form

$U(s)=\left[\begin{array}{llll}u_{1}(s) & u_{2}(s) & \ldots & u_{n}(s)\end{array}\right]^{\mathrm{T}}$

where

$u_{i}(s)=H_{i}(s) y_{i}(s)$

In eqn. (4), $y_{i}(s)$ is the output signal of generator $i$ and also the input signal to the PSS in generator $i$. Three types of input signal $y_{i}$ are usually employed, namely, speed, power and frequency $[6,7]$. The transfer function of a PI PSS, $H_{i}(s)$, is given by [24]

$H_{i}(s)=\frac{s}{1+s T_{i}}\left(K_{\mathrm{P} i}+\frac{K_{\mathrm{I} i}}{s}\right)$

The first factor on the right of (5) is a washout term while $K_{\mathrm{p}_{i}}$ and $K_{\mathbf{I} i}$ are the gains of the proportional stabilizer and the integral stabilizer, respectively, of generator $i$. The washout time constant $T_{i}$ is in the range $0.1-50 \mathrm{~s}$ and is usually preselected according to the desired steady-state characteristic. In this paper, a value of $0.1 \mathrm{~s}$ is chosen for $T_{i}$. This essentially leaves the stabilizer gains $K_{\mathrm{P} i}$ and $K_{\mathrm{I} i}$ of the PI PSS to be selected such that the dynamic stability of the power system can be improved.

Combining eqns. (2), (3) and (4), we have the state equation of the closed-loop system 
$s X(s)=\mathrm{A} X(s)+\mathrm{BH}(s) Y(s)$

where

$$
\begin{aligned}
& \mathbf{H}(s)=\operatorname{diag}\left[H_{1}(s) \quad H_{2}(s) \quad \ldots \quad H_{n}(s)\right]
\end{aligned}
$$

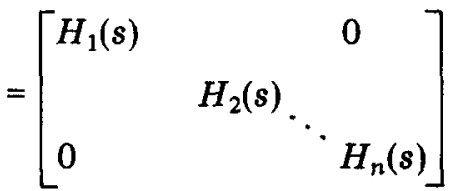

and

$Y(s)=\left[\begin{array}{llll}y_{1}(s) & y_{2}(s) & \ldots & y_{n}(s)\end{array}\right]^{\mathrm{T}}$

Since

$Y(s)=\mathrm{C}(s) X(s)$

we have

$s X(s)=\mathrm{A} X(s)+\mathrm{BH}(s) \mathrm{C}(s) X(s)$

or

$s X(s)=[\mathrm{A}+\mathrm{BH}(s) \mathrm{C}(s)] X(s)$

The eigenvalues of the system can be found by solving the characteristic equation

$\operatorname{det}[s \mathbf{I}-[\mathbf{A}+\mathbf{B H}(s) \mathbf{C}(s)]]=0$

where 'det' signifies the determinant of a matrix.

In order to enhance the dynamic stability of the system, it is desirable to assign $m$ $(m \leqslant n)$ pairs of its dominant eigenvalues to preselected locations in the $s$-plane by incorporating $m$ power system stabilizers into the system with their $2 m$ unknown parameters $\left(K_{\mathrm{P} i}, K_{\mathrm{I} i}, i=1,2, \ldots, m\right)$ to be determined from the solutions of a set of $2 \mathrm{~m}$ algebraic equations derived from the assignment of $m$ pairs of eigenvalues $[15,21-23,25,26]$. In electric power systems, the dominant eigenvalues to be shifted are those associated with the poorly damped electromechanical oscillation modes [1, 15, $21 \cdot 23]$.

It should be noticed that there are $2 m$ nonnumeric constants in matrix $H(s)$. Therefore, it would be very difficult to find the determinant on the right of (11) by a digital computer. In fact, this is the reason why a sequential algorithm [18] or an iterative algorithm [16 - 18, $21-23]$ was employed in previous works.

The principal contribution of this paper is to present a novel approach to find the $2 \mathrm{~m}$ algebraic equations directly in a very efficient way. The most important feature of the proposed method is to perform exact model reduction based on decentralized modal control (DMC) theory. The details of this approach will be discussed in the following section.

\section{AN EFFICIENT COORDINATED TUNING ALGORITHM}

Although the following discussions will be concentrated on the design of a PSS with the speed signal as its input, the proposed method can be applied to the tuning of PSSs with any kind of input signal.

It has been mentioned that $2 m$ eigenvalues associated with the $m$ poorly damped electromechanical modes will be shifted to $2 \mathrm{~m}$ preselected locations by the installation of PI stabilizers on $m$ generators. It is assumed, without any loss in generality, that generators $1,2, \ldots, m$ will be equipped with PSSs.

It is well known that electromechanical modes are the modes associated with rotor oscillations which are described by the state variables $\Delta \delta_{i}$ or $\Delta \omega_{i} \quad(i=1,2, \ldots, n)$. In order to fully characterize these rotor oscillations, it is sufficient to retain the speed deviations $\Delta \omega_{i}(i=1,2, \ldots, n)$ in the model reduction process since the deviations in rotor angle and angular speed are closely related to each other by the equation

$\Delta \omega_{i}=\frac{\mathrm{d}}{\mathrm{d} t} \Delta \delta_{i}$

Computer simulation results in $\$ 4$ will again illustrate this point.

Now, suppose each generator is described by five state variables, $\Delta \omega_{i}, \Delta \delta_{i}, \Delta e^{\prime}{ }_{\mathrm{q} i}, \Delta e_{\mathrm{FD} i}$ and $\Delta V_{F i}[1]$, then the state equation (2) can be rewritten as

$\left[\begin{array}{c}s X_{1}(s) \\ \hdashline s X_{2}(s)\end{array}\right]=\left[\begin{array}{c:c}\mathrm{A}_{11} & \mathrm{~A}_{12} \\ \hdashline \mathrm{A}_{21} & \mathrm{~A}_{22}\end{array}\right]\left[\begin{array}{c}X_{1}(s) \\ \hdashline X_{2}(s)\end{array}\right]+\left[\begin{array}{c}\mathrm{B}_{1} \\ \hdashline \mathrm{B}_{2}\end{array}\right] U(s)$

where

$$
\begin{aligned}
X_{1}(s)= & {\left[\Delta \omega_{1}(s), \Delta \omega_{2}(s), \ldots, \Delta \omega_{i}(s),\right.} \\
& \left.\ldots, \Delta \omega_{n}(s)\right]^{\mathrm{T}} \\
X_{2}(s)= & {\left[\Delta \delta_{1}(s), \ldots, \Delta \delta_{n}(s), \Delta e^{\prime}{ }_{\mathrm{q} 1}(s),\right.} \\
& \ldots, \Delta e_{\mathrm{q} n}^{\prime}(s), \Delta e_{\mathrm{FD} 1}(s), \\
& \left.\ldots, \Delta e_{\mathrm{FD} n}(s), \Delta V_{\mathrm{F} 1}(s), \ldots, \Delta V_{\mathrm{F} n}(s)\right]^{\mathrm{T}}
\end{aligned}
$$


Since $y_{i}(s)=\Delta \omega_{i}(s)$ for $i=1,2, \ldots, n$, we have $Y(s)=X_{1}(s)$

Then, from eqn. (6), the control vector $U(s)$ and the matrices $\mathbf{B}_{1}$ and $\mathbf{B}_{2}$ can be written as

$$
\begin{aligned}
U(s)= & \mathbf{H}(s) X_{1}(s) \\
= & {\left[H_{1}(s) \Delta \omega_{1}(s), H_{2}(s) \Delta \omega_{2}(s),\right.} \\
& \left.\ldots, H_{n}(s) \Delta \omega_{n}(s)\right]^{\mathrm{T}} \\
\mathbf{B}_{1}= & \mathbf{O}_{(n \times n)}
\end{aligned}
$$

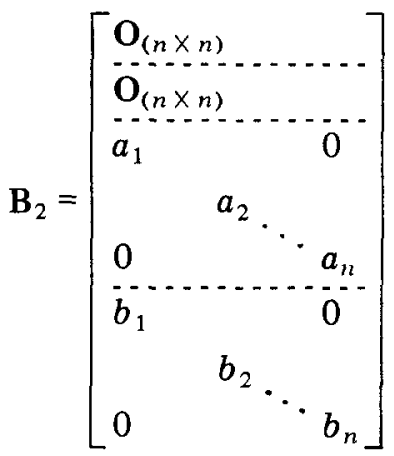

where

$$
\begin{array}{ll}
a_{i}=\frac{K_{\mathrm{A} i}}{T_{\mathrm{A} i}} & i=1, \ldots, n \\
b_{i}=\frac{K_{\mathrm{F} i} K_{\mathrm{A} i}}{T_{\mathrm{F} i} T_{\mathrm{A} i}} & i=1, \ldots, n
\end{array}
$$

It should be noted that the transfer function matrix $\mathbf{H}(s)$ in eqn. (15) has been defined in eqn. (7). Furthermore, $H_{i}(s)=0$ for $i=m+1$, $\ldots, n$ because only $m$ generators will be equipped with PSSs.

Using eqns. (15), (16) and (17), we can rewrite eqn. (13) as

$$
\begin{aligned}
{\left[\begin{array}{l}
s X_{1}(s) \\
\hdashline s X_{2}(s)
\end{array}\right]=} & {\left[\begin{array}{ccc}
\mathbf{A}_{11} & \mathbf{A}_{12} \\
\hdashline \mathbf{A}_{21} & \mathbf{A}_{22}
\end{array}\right]\left[\begin{array}{l}
X_{1}(s) \\
\hdashline X_{2}(s)
\end{array}\right] } \\
& +\left[\begin{array}{c}
\mathbf{O}_{(n \times n)} \\
\hdashline \mathbf{B}_{2}^{\prime}(s)
\end{array}\right] X_{1}(s)
\end{aligned}
$$

where

$$
\begin{aligned}
& B^{\prime}{ }_{2}(s)=\mathbf{B}_{2} \mathbf{H}(s)
\end{aligned}
$$

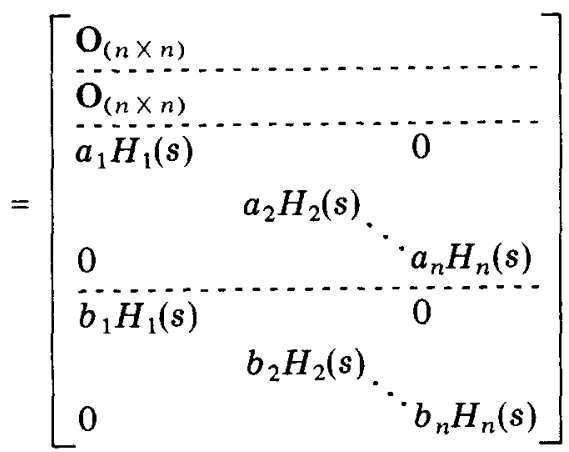

It follows that

$$
\left[\begin{array}{l}
s X_{1}(s) \\
\hdashline s X_{2}(s)
\end{array}\right]=\left[\begin{array}{l:c}
\mathrm{A}_{11} & \mathrm{~A}_{12} \\
\hdashline \mathrm{A}_{21}+\mathrm{B}_{2}(s) & \mathrm{A}_{22}
\end{array}\right]\left[\begin{array}{c}
X_{1}(s) \\
\hdashline X_{2}(s)
\end{array}\right]
$$

or

$$
\begin{aligned}
& s X_{1}(s)=\mathrm{A}_{11} X_{1}(s)+\mathrm{A}_{12} X_{2}(s) \\
& X_{2}(s)=\left(s \mathrm{I}-\mathrm{A}_{22}\right)^{-1}\left[\mathrm{~A}_{21}+\mathrm{B}^{\prime}{ }_{2}(s)\right] X_{1}(s)
\end{aligned}
$$

Substituting eqn. (25) into (24), we get

$$
\begin{aligned}
& \left\{\left[s \mathbf{I}-\mathbf{A}_{11}-\mathbf{A}_{12}\left(s \mathbf{I}-\mathbf{A}_{22}\right)^{-1} \mathbf{A}_{21}\right]\right. \\
& \left.\quad-\mathbf{A}_{12}\left(s \mathbf{I}-\mathbf{A}_{22}\right)^{-1} \mathbf{B}^{\prime}{ }_{2}(s)\right\} X_{1}(s) \\
& =0
\end{aligned}
$$

Let $\lambda$ be an eigenvalue associated with the electromechanical modes which dominate the vector $X_{1}(s)$ comprising the rotor speed deviations of the $n$ generators. Then, we have

$\operatorname{det}\left[\mathbf{R}(\lambda)-\mathbf{P}(\lambda) \mathbf{B}_{2}^{\prime}(\lambda)\right]=0$

where

$\mathbf{R}(\lambda)=\left[\lambda \mathbf{I}-\mathbf{A}_{11}-\mathbf{A}_{12}\left(\lambda \mathbf{I}-\mathbf{A}_{22}\right)^{-1} \mathbf{A}_{21}\right]_{(n \times n)}$

$\mathbf{P}(\lambda)=\left[\mathbf{A}_{12}\left(\lambda \mathbf{I}-\mathbf{A}_{22}\right)^{-1}\right]_{(n \times n)}$

If we let

$\mathbf{P}^{\prime}(\lambda)=-\mathbf{P}(\lambda) \mathbf{B}_{2}^{\prime}(\lambda)$

and substitute eqn. (22) into (30), we can express $\mathbf{P}_{i}^{\prime}$, the $i$ th column of the matrix $\mathbf{P}^{\prime}(\lambda)$, as

$\mathbf{P}^{\prime}{ }_{i}=-\left[H_{i}(\lambda)\left(a_{1} \mathbf{P}_{2 n+i}+b_{i} \mathbf{P}_{3 n+i}\right)\right]$

or

$\mathbf{P}_{i}^{\prime}=H_{i}(\lambda) \mathbf{P}_{i}^{\prime \prime}$

where

$\mathbf{P}_{2 n+i}=(2 n+i)$ th column of $\mathbf{P}(\lambda)$

$\mathbf{P}_{3 n+i}=(3 n+i)$ th column of $\mathbf{P}(\lambda)$

and

$\mathbf{P}_{i}^{\prime \prime}=-a_{i} \mathbf{P}_{2 n+i}-b_{i} \mathbf{P}_{3 n+i}$

is the $i$ th column of matrix $\mathbf{P}^{\prime \prime}(\lambda)$.

Substituting eqn. (30) into (27), we have

$\operatorname{det}\left[\mathbf{R}(\lambda)+\mathbf{P}^{\prime}(\lambda)\right]=0$

or

$$
\begin{aligned}
\operatorname{det} & {\left[\mathbf{R}_{1}+\mathbf{P}_{1}^{\prime}, \mathbf{R}_{2}+\mathbf{P}^{\prime}{ }_{2}, \ldots, \mathbf{R}_{i}+\mathbf{P}^{\prime}{ }_{i},\right.} \\
& \left.\ldots, \mathbf{R}_{n}+\mathbf{P}^{\prime}{ }_{n}\right] \\
= & 0
\end{aligned}
$$

where $\mathbf{R}_{\boldsymbol{i}}$ is the ith column of $\mathbf{R}(\lambda)$. 
Finally, using the following linearity property of the determinants of matrices,

$$
\begin{aligned}
& \operatorname{det} {\left[\mathbf{A}_{1}, \mathbf{A}_{2}, \ldots, \mathbf{A}_{i}+k \mathbf{A}_{i}^{\prime}, \ldots, \mathbf{A}_{n}\right] } \\
& \quad=\operatorname{det}\left[\mathbf{A}_{1}, \mathbf{A}_{2}, \ldots, \mathbf{A}_{i}, \ldots, \mathbf{A}_{n}\right]+k \operatorname{det}\left[\mathbf{A}_{1}, \mathbf{A}_{2}, \ldots, \mathbf{A}_{i}^{\prime}, \ldots, \mathbf{A}_{n}\right]
\end{aligned}
$$

we can get

$$
\begin{aligned}
& \operatorname{det}[\mathbf{R}(\lambda)]+\sum_{\substack{m_{1}, m_{2}=1, \ldots, n \\
m_{1} \neq m_{2}}} d_{m_{1} m_{2}} H_{m_{1}}(\lambda) H_{m_{2}}(\lambda) \\
& \quad+\sum_{\substack{m_{1}, m_{2}, m_{3}=1, \ldots, n \\
m_{1} \neq m_{2} \neq m_{3}}} d_{m_{1} m_{2} m_{3}} H_{m_{1}}(\lambda) H_{m_{2}}(\lambda) H_{m_{3}}(\lambda) \\
& \quad+\ldots+\sum_{\substack{m_{1}, \ldots, m_{k}=1, \ldots, n \\
m_{1} \neq \ldots \neq m_{k}}} d_{m_{1} m_{2} \ldots m_{k}} H_{m_{1}}(\lambda) H_{m_{2}}(\lambda) \ldots H_{m_{k}}(\lambda) \\
& \quad+\ldots+\sum_{\substack{m_{1} \ldots, \ldots m_{n}=1, \ldots, n \\
m_{1} \neq \ldots \neq m_{n}}} d_{m_{1} m_{2} \ldots m_{n}} H_{m_{1}}(\lambda) H_{m_{2}}(\lambda) \ldots H_{m_{n}}(\lambda) \\
& =0
\end{aligned}
$$

where $d_{m_{1} m_{2} \ldots m_{k}}$ is the determinant of a matrix which is the same as the matrix $\mathbf{R}(\lambda)$ except that its $m_{1}$ th, $m_{2}$ th, ..., and $m_{k}$ th columns are replaced by the corresponding columns of matrix $P^{\prime \prime}(\lambda)$. Equation (36) can be expressed in a more compact form:

$\operatorname{det}[\mathbf{R}(\lambda)]+\sum_{k=1}^{n}\left[\sum_{\substack{m_{1}, m_{2} \ldots, m_{k}=1, \ldots, n \\ m_{1} \neq m_{2} \neq \ldots \neq m_{k}}} d_{m_{1} m_{2} \ldots m_{k}} H_{m_{1}}(\lambda) H_{m_{2}}(\lambda) \ldots H_{m_{k}}(\lambda)\right]=0$

When the $2 m$ assigned eigenvalues associated with the electromechanical oscillation modes are substituted into eqn. (37), we obtain the $2 m$ equations required for the simultaneous determination of the $2 m$ unknown PSS parameters $K_{\mathrm{P} 1}, K_{\mathrm{I} 1}, K_{\mathrm{P} 2}, K_{\mathrm{I} 2}, \ldots, K_{\mathrm{P} m}, K_{1 m}$. These equations can be written as

$f_{1}\left(K_{\mathrm{P} 1}, K_{\mathrm{I} 1}, K_{\mathrm{P} 2}, K_{\mathrm{I} 2}, \ldots, K_{\mathrm{P} m}, K_{\mathrm{I} m}\right)=0$

$f_{2}\left(K_{\mathrm{P} 1}, K_{\mathrm{I} 1}, K_{\mathrm{P} 2}, K_{\mathrm{I} 2}, \ldots, K_{\mathrm{P} m}, K_{\mathrm{Im}}\right)=0$

$f_{2 m}\left(K_{\mathrm{P} 1}, K_{\mathrm{I} 1}, K_{\mathrm{P} 2}, K_{\mathrm{I} 2}, \ldots, K_{\mathrm{P} m}, K_{\mathrm{l} m}\right)=0$

or, more compactly,

$f_{i}\left(K_{\mathrm{P} 1}, K_{11}, K_{\mathrm{P} 2}, K_{12}, \ldots, K_{\mathrm{P} m}, K_{\mathrm{I} m}\right)=0 \quad i=1,2, \ldots, 2 m$

In order to find the PSS parameters $K_{\mathrm{P} i}$ and $K_{I i}(i=1,2, \ldots, m)$, the well-known NewtonRaphson method can be employed to solve eqn. (39). Figure 1 gives the flow chart for the proposed algorithm.

\section{EXAMPLE 1 : SINGLE-MACHINE-INFINITE-BUS SYSTEM}

Consider a single-machine-infinite-bus system whose linearized incremental model including the voltage regulator and exciter can be represented by the block diagram shown in Fig. 2 [3, 4, $6,7]$. The parameters of the system are given as follows [24]: 


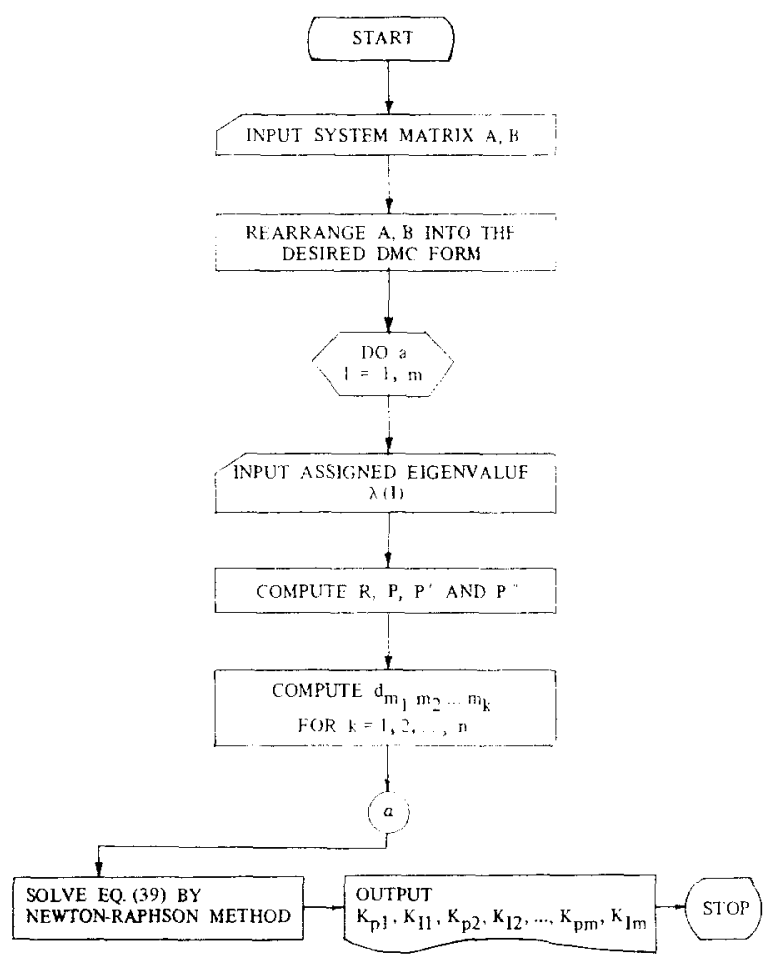

Fig. 1. Flow chart of the DMC algorithm.

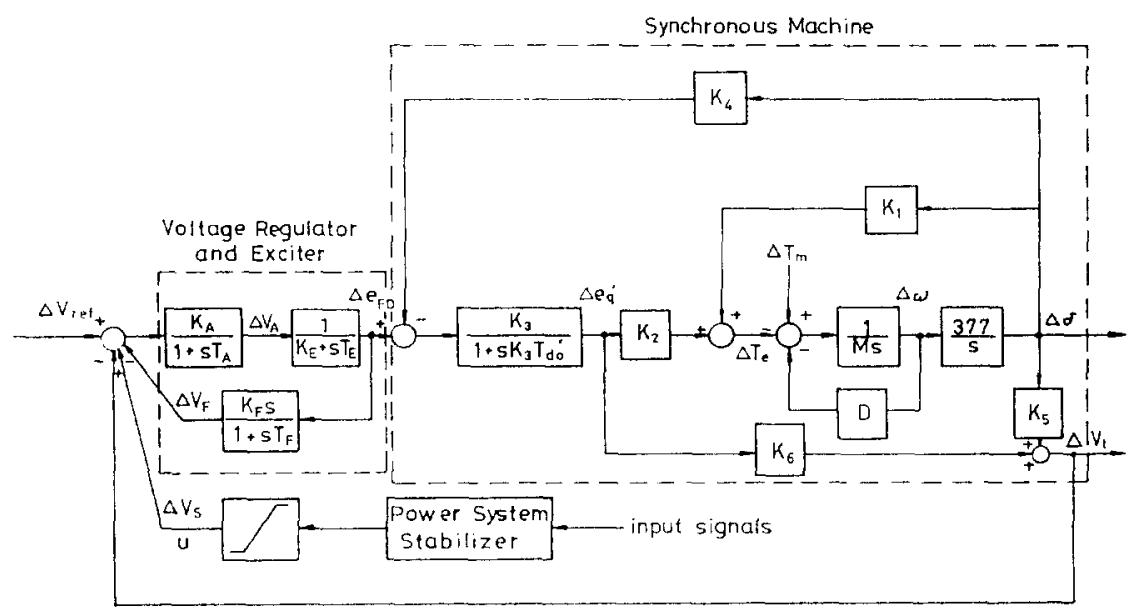

Fig. 2. Linearized incremental model of synchronous machine with an exciter and stabilizer.

$\begin{array}{lll}K_{1}=1.4479 & K_{\mathrm{A}}=400 & D=0 \\ K_{2}=1.3174 & T_{\mathrm{A}}=0.05 & T_{\mathrm{d} 0}^{\prime}=5.9 \\ K_{3}=0.3072 & K_{\mathrm{E}}=-0.17 & M=4.74 \\ K_{4}=1.8050 & T_{\mathrm{E}}=0.95 & \\ K_{5}=0.0294 & K_{\mathrm{F}}=0.025 & \\ K_{6}=0.5257 & T_{\mathrm{F}}=1.0 & \end{array}$

Then the corresponding state equation can be written in the form $\dot{X}=\mathbf{A} X+\mathbf{B} U$ 
where

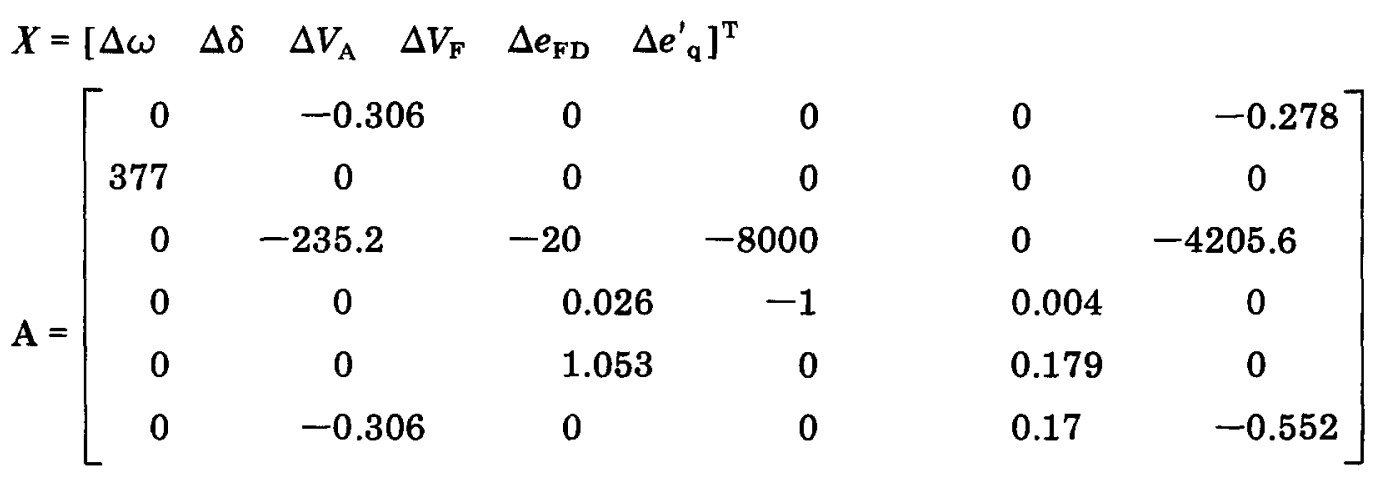

$B=\left[\begin{array}{llllll}0 & 0 & 8000 & 0 & 0 & 0\end{array}\right]^{\mathrm{T}}$

The eigenvalues of the open-loop system without PSS are listed in the first column of Table 1. It is clear from this Table that damping of the electromechanical mode $(-0.235 \pm j 10.8)$ is not enough from the viewpoint of dynamic stability. Therefore, a PSS has to be added to the synchronous generator in order to enhance the damping of the system.

As mentioned in $\$ 2$, the transfer function of the PI stabilizer is given by

$H(s)=\frac{s}{1+0.1 s}\left(K_{\mathrm{P}}+\frac{K_{\mathrm{I}}}{s}\right)$

Using eqn. (39), the parameters of the PI stabilizer are calculated as

$K_{\mathrm{P}}=-1.075, \quad K_{\mathrm{I}}=-15.528$

The eigenvalues of the closed-loop system with PSS incorporated are given in the second column of Table 1. It can be concluded from this Table that exact assignment of the eigenvalues associated with the electromechanical mode is achieved without eigenvalue drift.

TABLE 1

Eigenvalues for the single-machine system

\begin{tabular}{ll}
\hline Open loop (without PSS) & $\begin{array}{l}\text { Closed loop (with PSS) } \\
(\zeta=0.2)\end{array}$ \\
\hline$-0.235 \pm \mathrm{j} 10.8$ & $-2.16 \pm \mathrm{j} 10.73^{\mathrm{a}}$ \\
$-8.13 \pm \mathrm{j} 8.98$ & $-4.78 \pm \mathrm{j} 6.33$ \\
-3.1 & $-8.04 \pm \mathrm{j} 3.59$ \\
-1.5 & -1.4 \\
\hline
\end{tabular}

${ }^{a}$ Denotes exact assignment of electromechanical-mode eigenvalues.

Figure 3 shows the dynamic responses of $\Delta \delta$ and $\Delta \omega$ when the system is subjected to a 0.05 p.u. step change in the mechanical torque $\Delta T_{\mathrm{m}}$. The responses for the system without and with PSS are both included for comparison purposes. It can be seen from Fig. 3 that the damping characteristic of the system can be significantly improved by the PSS. Moreover, even though our design process is concentrated primarily on $\Delta \omega$, the damping of $\Delta \delta$ can be equally as enhanced as that of $\Delta \omega$. This is due to the fact that $\Delta \delta$ and $\Delta \omega$ are closely related by eqn. (12).

\section{EXAMPLE 2 : MULTMMACHINE POWER SYSTEM}

Consider a three-machine-infinite-bus system $[15,22,23]$. The system configuration is shown in Fig. 4 and the system data are given in Table 2. 


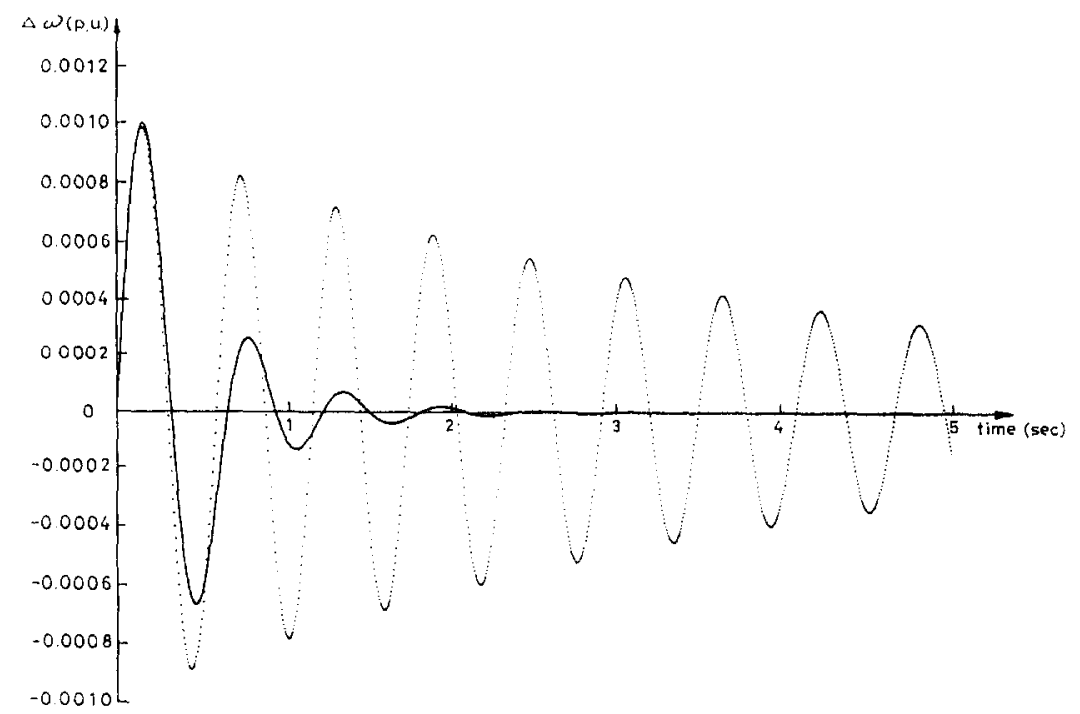

(a)

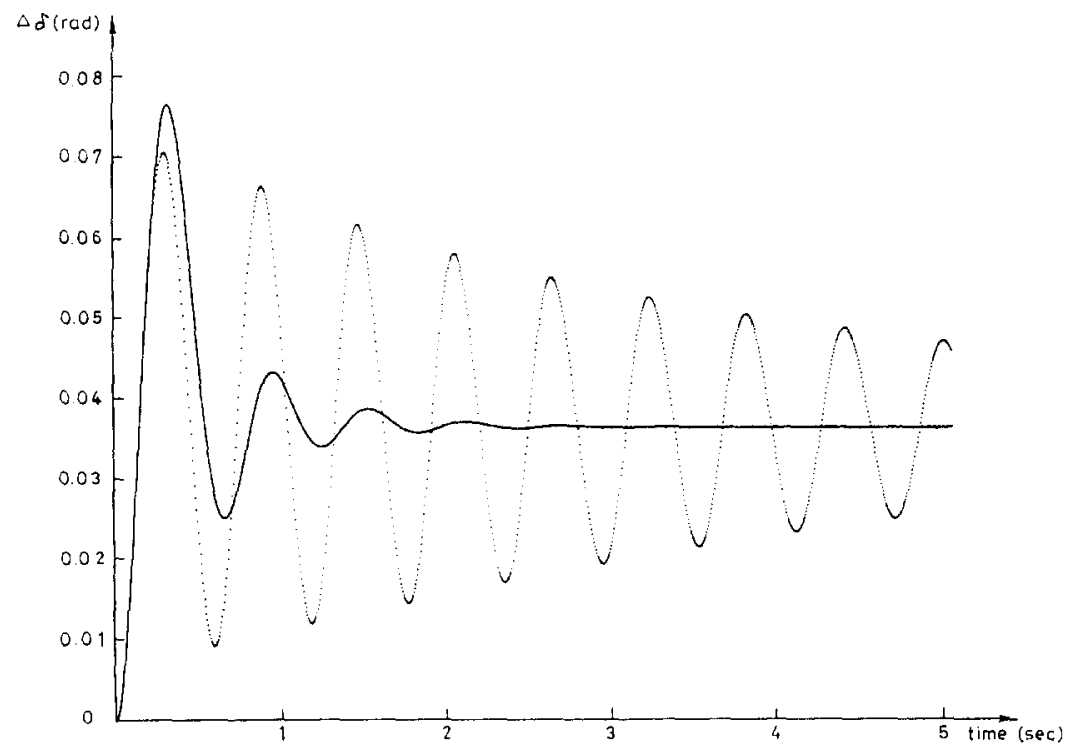

(b)

Fig. 3. Dynamic responses for a 0.05 p.u. step change in $\Delta T_{\mathrm{m}}(\cdots$, , without PSS; —-, with PSS): (a) deviation in angular speed; (b) deviation in torque angle.

Each plant is represented by a third-order generator equipped with a static exciter. The state equation of this system is given by

$\dot{X}=\mathbf{A} \boldsymbol{X}+\mathbf{B} \boldsymbol{U}$

where

$X=\left[\begin{array}{llllllllllll}\Delta \omega_{1} & \Delta \omega_{2} & \Delta \omega_{3} & \Delta \delta_{1} & \Delta \delta_{2} & \Delta \delta_{3} & \Delta e_{\mathrm{q} 1}^{\prime} & \Delta e_{\mathrm{q} 2}^{\prime} & \Delta e_{\mathrm{q} 3}^{\prime} & \Delta e_{\mathrm{FD} 1} & \Delta e_{\mathrm{FD} 2} & \Delta e_{\mathrm{FD} 3}\end{array}\right]^{\mathrm{T}}$ is the state vector and

$U=\left[\begin{array}{lll}u_{1} & u_{2} & u_{3}\end{array}\right]^{\mathbf{T}}$

is the input vector. The system matrices A and B are given in eqns. (46) and (47): 


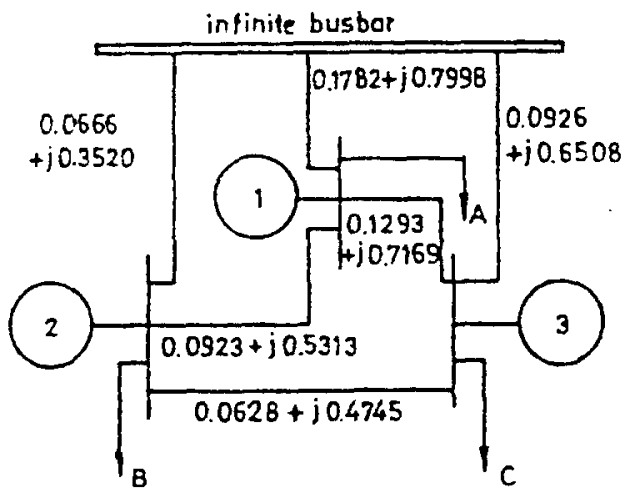

Fig. 4. Three-machine-infinite-bus system.

TABLE 2

The multimachine power system data

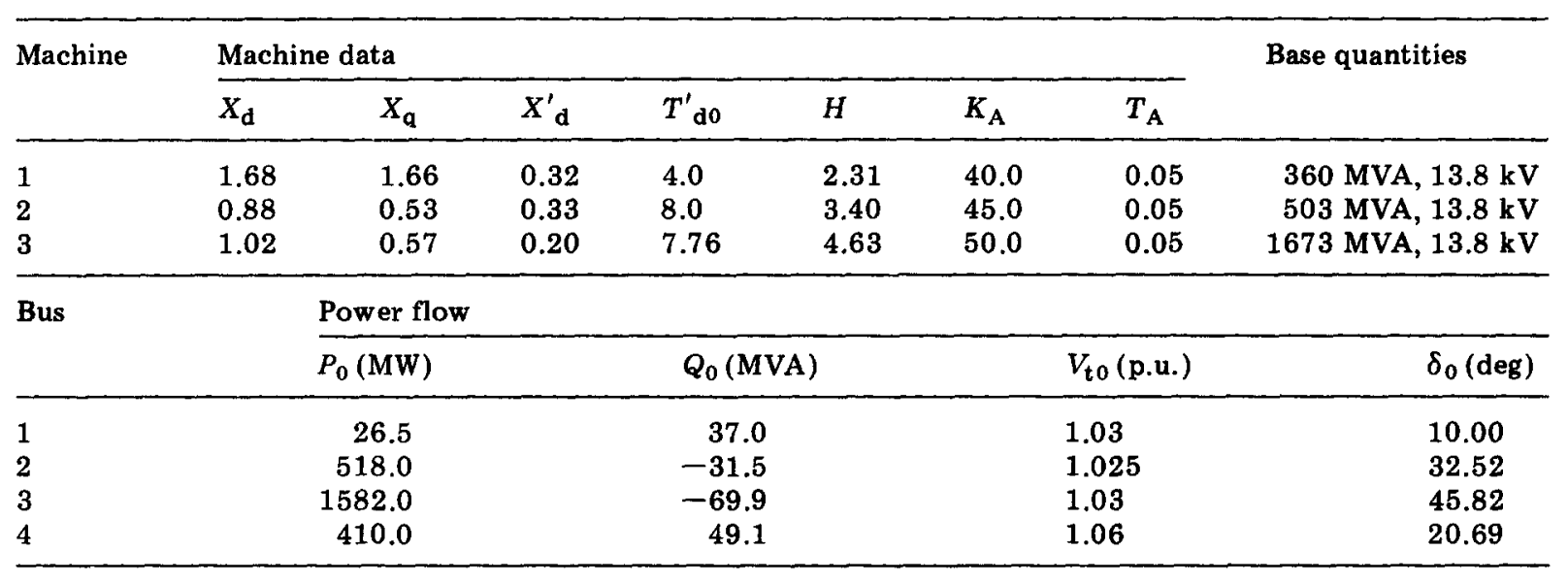

$\mathbf{A}=$

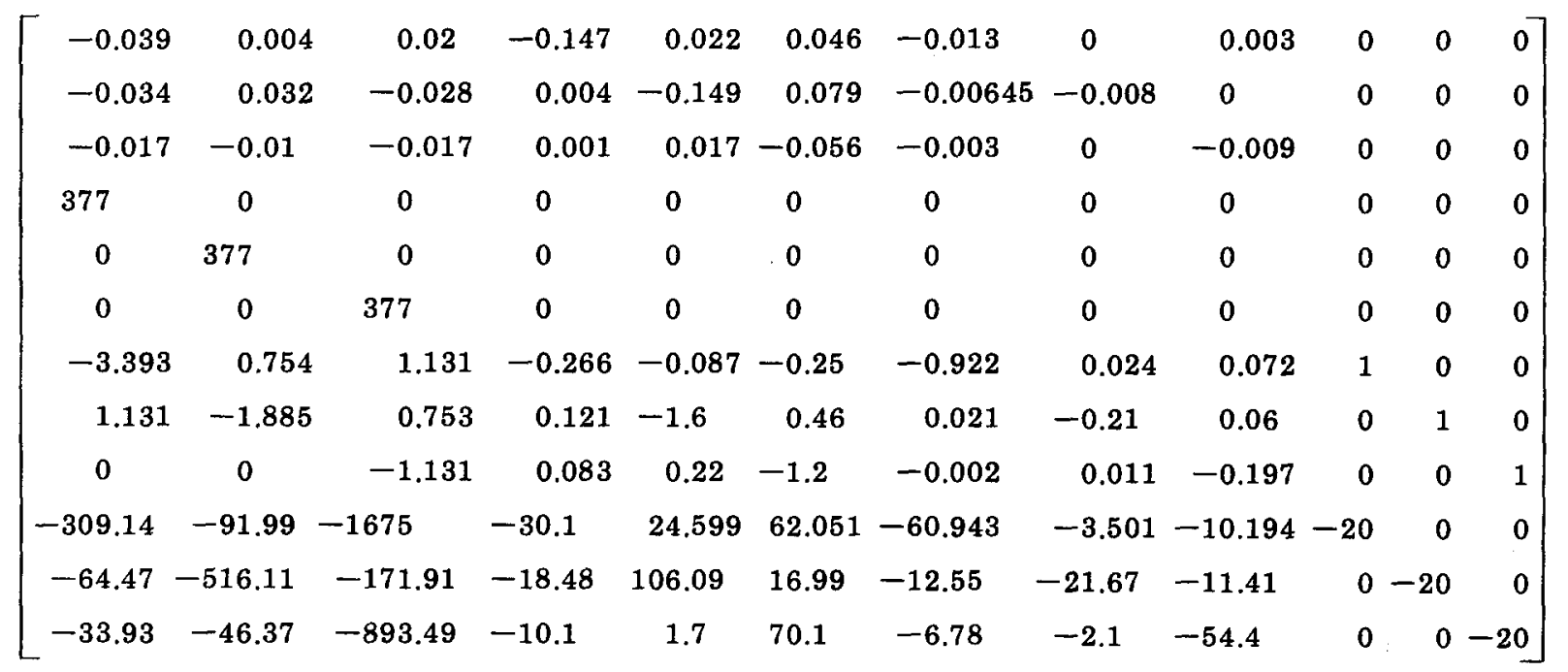

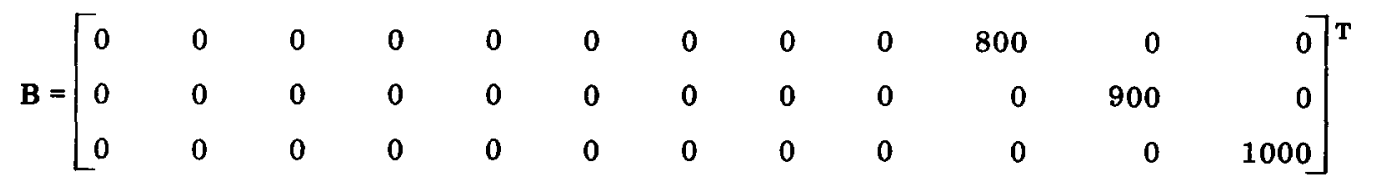


80
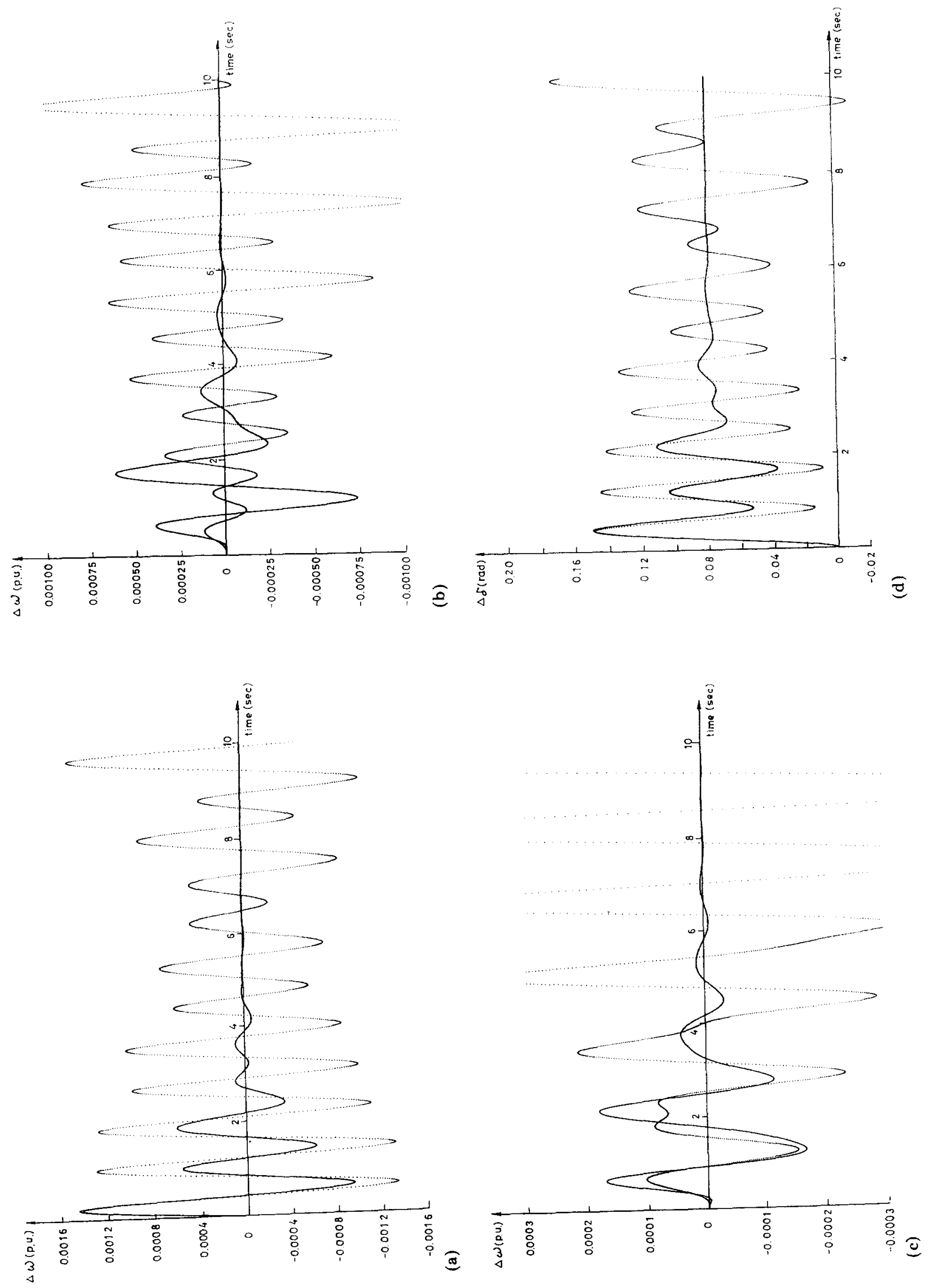


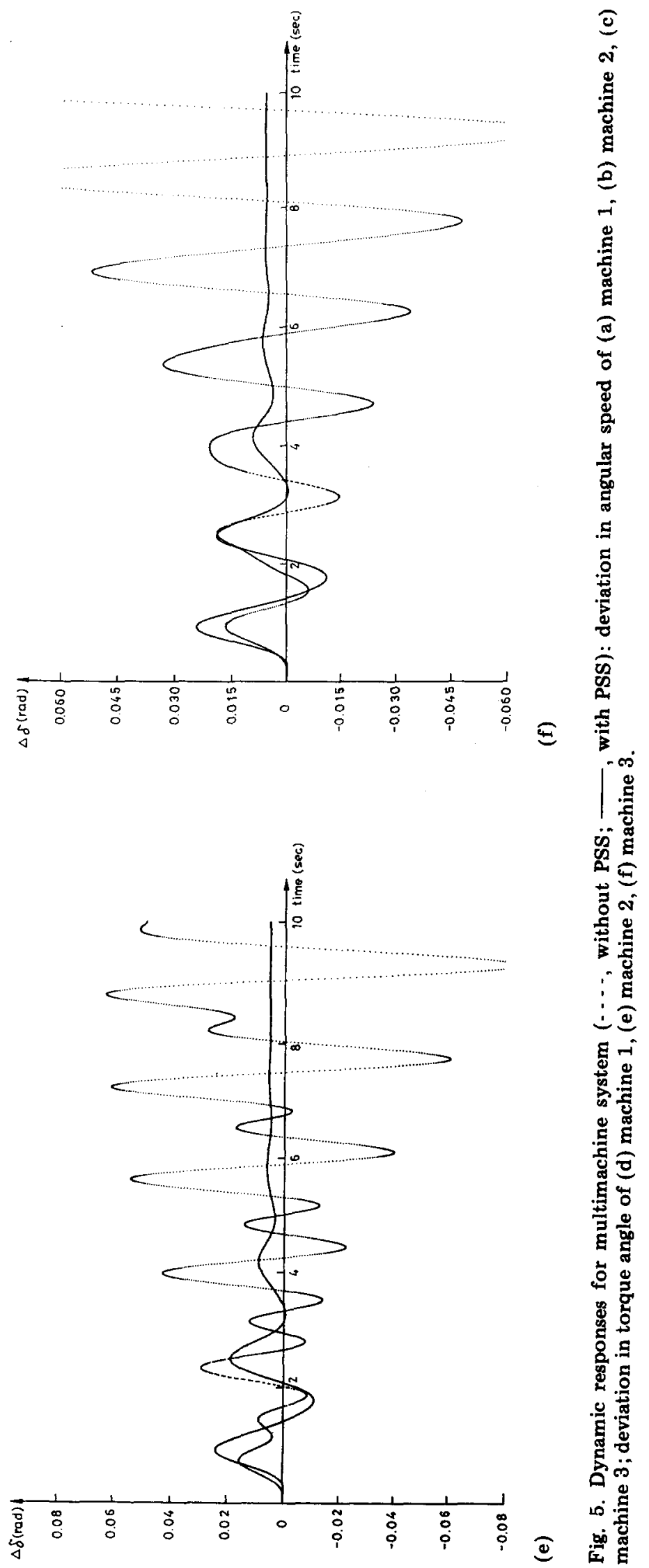


The eigenvalues of the system without PSS are listed in the first column of Table 3 . It can be seen from this Table that the open-loop system is unstable and three PI stabilizers have to be installed on the three generators in order to shift the three pairs of electromechanical-mode eigenvalues with poor damping to the desired locations.

Using eqn. (39), we can compute the desired stabilizer constants $K_{\mathrm{P}_{i}}$ and $K_{\mathrm{I} i}(i=$ $1,2,3)$ and the closed-loop eigenvalues of the system as shown in the second column of Table 3.

Figure 5 gives the dynamic responses of $\Delta \omega$ and $\Delta \delta$ for the multimachine system when machine 1 is subjected to a 0.05 p.u. step change in mechanical torque $\Delta T_{\mathrm{m}}$.

\section{TABLE 3}

Eigenvalues and the computed stabilizer parameters for the multimachine power system

\begin{tabular}{lcc}
\hline & $\begin{array}{l}\text { Open loop } \\
\text { (without PSS) }\end{array}$ & $\begin{array}{l}\text { Closed loop } \\
\text { (with PSS) } \\
\zeta=0.2\end{array}$ \\
\hline Stabilizer & & \\
parameters & & \\
$K_{\mathrm{P} 1}$ & & 2.556 \\
$K_{11}$ & & -22.01 \\
$K_{\mathrm{P} 2}$ & & -8.996 \\
$K_{\mathrm{I} 2}$ & & -75.92 \\
$K_{\mathrm{P} 3}$ & & 12.78 \\
$K_{\mathrm{I} 3}$ & & 9.191 \\
Eigenvalues & $-0.0627 \pm \mathrm{j} 7.37$ & $-1.47 \pm \mathrm{j} 7.35^{\mathrm{a}}$ \\
& $0.0953 \pm \mathrm{j} 7.84$ & $-1.38 \pm \mathrm{j} 6.9^{\mathrm{a}}$ \\
& $0.264 \pm \mathrm{j} 4.09$ & $-0.76 \pm \mathrm{j} 3.8^{\mathrm{a}}$ \\
& -1.51 & $-4.79 \pm \mathrm{j} 5.5$ \\
& -3.43 & $-1.86 \pm \mathrm{j} 3.88$ \\
& -5.89 & -2.66 \\
& -15.19 & -11.82 \\
& -17.05 & -15.63 \\
& -18.87 & -19.28 \\
& & -21.44 \\
\hline
\end{tabular}

${ }^{a}$ Denotes exact assignment of electromechanicalmode eigenvalues.

\section{DISCUSSION}

From the examples in $\S \S 4$ and 5, several observations are in order.

(1) Exact assignment of any number of pairs of complex-conjugate eigenvalues associ- ated with the poorly damped electromechanical modes of oscillation can be achieved by simultaneously selecting the PI PSS parameters using the proposed DMC algorithm.

(2) In the proposed DMC algorithm, the parameters of PI stabilizers are obtained by directly solving a set of algebraic equations. Hence, the DMC algorithm is superior to the iterative algorithm employed in previous works from the viewpoint of computational effort. The DMC algorithm is highly efficient in the coordinated tuning of PI PSS parameters. For the multimachine power system considered in $\S 5$, the required CPU time on the VAX-11/780 computer is only about $1.4 \mathrm{~s}$.

(3) As in the case of the power flow problem, the PSS parameters obtained from the solution of a set of nonlinear equations in the DMC algorithm will not be unique. Any set of stabilizer parameters within the tuning range of the PSS will be acceptable. Usually, it is desirable to choose a set of parameters with the smallest PSS gain since a PSS with large gain will lead to a deterioration of the terminal voltage profile.

(4) In contrast to the iterative algorithms in the previous works, no convergence problem would result in the proposed DMC algorithm since it is not iterative in nature.

\section{CONCLUSIONS}

In this paper, a new method for the coordinated tuning of the parameters of proportional-integral power system stabilizers in multimachine power systems is presented. Exact model reduction based on decentralized modal control (DMC) is employed to make the proposed algorithm highly efficient. The method is novel in that the stabilizer parameters are computed directly by solving a set of algebraic equations obtained by the exact assignment of several eigenvalues associated with the poorly damped electromechanical modes to preselected locations. Hence, it is much more efficient than the iterative algorithms used in previous works. The proposed algorithm can be extended easily to the design of PSSs with other types of input signals such as electric power. In doing so, the only change in the algorithm is to select the power as a state variable of the system. 


\section{REFERENCES}

1 C. Barbier, E. Ferrari and K. E. Johansson, Questionnaire on electromechanical oscillation damping in power systems: report on answers, Electra, 64 (1979) $59-90$.

2 E. V. Larsen and D. A. Swann, Applying power system stabilizers, IEEE Trans., PAS-100 (1981) $3017 \cdot 3046$.

3 W. C. Chan and Y. Y. Hsu, An optimal variable structure stabilizer for power system stabilization, IEEE Trans., PAS-102 (1983) 3017 - 3046.

4 F. P. de Mello, and C. Concordia, Concepts of synchronous machine stability as affected by excitation control, IEEE Trans., PAS-88 (1969) $316-329$.

5 F. R. Schleif, G. E. Martin and R. R. Angell, Damping of system oscillations with a hydrogenerating unit, IEEE Trans., PAS-86 (1967) $438-442$

6 P. M. Anderson and A. A. Fouad, Power System Control and Stability, Iowa State University Press, Ames, IA, 1977.

7 Y. N. Yu, Electric Power System Dynamics, Academic Press, New York, 1983.

8 O. W. Hanson, C. J. Goodwin and P. L. Dandeno, Influence of excitation and speed control param. eters in stabilizing intersystem oscillation, IEEE Trans., PAS-87 (1968) 1306 - 1313.

9 J. E. Van Ness, F. M. Brasch, Jr., G. L. Landgran and S. T. Naumann, Analytical investigation of dynamic instability occurring in Powerton station, IEEE Trans., PAS-99 (1980) 1386 - 1395.

$10 \mathrm{~W}$. Watson and G. Manchur, Experience with supplementary damping signals for generator static excitation systems, IEEE Trans., PAS-92 (1973) $199 \cdot 203$.

11 A. B. R. Kumar and E. F. Richards, An optimal control law by eigenvalue assignment for im. proved dynamic stability in power systems, IEEE Trans., PAS-101 (1982) 1570 - 1577.

12 A. Olwegard, Improvement of system stability in interconnected power systems, CIGRE, 1970, Paper No. $32-17$.

13 F. P. de Mello, P. J. Nolan, T. F. Laskowski and J. M. Undrill, Coordinated application of stabi- lizers in multimachine power systems, IEEE Trans., PAS.99 (1980) 892 - 901.

14 O. H. Abdalla, S. A. Hassan and N. T. Tweig, Coordinated stabilization of a multimachine power system, IEEE Trans., PAS-103 (1984) $483-494$.

15 R. J. Fleming, M. A. Mohan and K. Parvatisam, Selection of parameters of stabilizers in multimachine power systems, IEEE Trans., PAS-100 (1981) 2329 - 2333.

16 H. B. Gooi, E. F. Hill, M. A. Mobarak, D. H. Thorne and T. H. Lee, Coordinated multi-machine stabilizer settings without eigenvalue drift, IEEE Trans., PAS-100 (1981) 3879 - 3887.

$17 \mathrm{~S}$. Lefevbre, Tuning of stabilizers in multimachine power systems, IEEE Trans., PAS-102 (1983) $290-299$.

18 S. Sivakumar, A. M. Sharaf and H. G. Hamed, Coordinated tuning of power system stabilizers in multimachine power systems, Electr. Power Syst. Res., 8 (1985) 275 - 284.

$19 \mathrm{~S}$. Abe and A. Doi, A new power system stabilizer synthesis in multimachine power systems, IEEE Trans., PAS-102 (1983) 3910 - 3918.

$20 \mathrm{~A}$. Doi and S. Abe, Coordinated synthesis of power system stabilizers in multimachine power systems, IEEE Trans., PAS-103 (1984) 1473 . 1479.

21 C. M. Lim and L. Elangovan, New approach to power system stabilizer design, Electr. Power Syst. Res., 8 (1985) 285 - 292.

22 C. M. Lim and L. Elangovan, Design of stabilizers in multimachine power systems, Proc. Inst. Electr. Eng., Part C, 132 (1985) 146 - 153.

23 C. M. Lim and L. Elangovan, A new stabilizer design technique for multimachine power systems, IEEE Trans., PAS-104 (1985) $2393-2400$.

24 Y. Y. Hsu and C. Y. Hsu, Design of a proportionalintegral power system stabilizer, IEEE PES Summer Meeting, Vancouver, Canada, 1985, Paper No. 85 SM 470-0.

25 I. J. Perez-Arriaga, G. C. Verghese and F. C. Schweppe, Selective modal analysis with applications to electric power systems, IEEE Trans., PAS-101 (1982) 3117 - 3134.

26 B. Porter and R. Crossley, Modal Control Theory and Applications, Taylor and Francis, London, 1972. 